Mathematical Modelling and Analysis

Volume 20 Number 2, March 2015, 273-288

http://dx.doi.org/10.3846/13926292.2015.1022236

(c) Vilnius Gediminas Technical University, 2015
Publisher: Taylor\&Francis and VGTU

http://www.tandfonline.com/TMMA

ISSN: $1392-6292$

eISSN: $1648-3510$

\title{
A Pair of Perfectly Conducting Disks in an External Field
}

\section{Natalia Rylko}

\author{
Institute of Technology, Pedagogical University of Cracow \\ ul. Podchorazych 2, 30-084 Krakow, Poland \\ E-mail: nrylko@up.krakow.pl
}

Received August 28, 2014; revised February 11, 2015; published online March 15, 2015

\begin{abstract}
A pair of non-overlapping perfectly conducting equal disks embedded in a two-dimensional background was investigated by the classic method of images, by Poincaré series, by use of the bipolar coordinates and by the elliptic functions in the previous works. In particular, successive application of the inversions with respect to circles were applied to obtain the field in the form of a series. For closely placed disks, the previous methods yield slowly convergent series. In this paper, we study the local fields around closely placed disks by the elliptic functions. The problem of small gap is completely investigated since the obtained closed form solution admits a precise asymptotic investigation in terms of the trigonometric functions when the gap between the disks tends to zero. The exact and asymptotic formulae are extended to the case when a prescribed singularity is located in the gap. This extends applications of structural approximations to estimations of the local fields in densely packed fiber composites in various external fields.
\end{abstract}

Keywords: perfectly conducting disks, small gap problem, boundary value problem.

AMS Subject Classification: $30 \mathrm{E} 25$.

\section{Introduction}

The famous Villat-Dini formula [12,31] (see [2]) solves the Dirichlet problem for a circular annulus $R^{-1}<|w|<R(R>1)$ in terms of the Poisson type integral with the kernel expressed through the elliptic functions. An alternative formula via the Fourier series was also known. Though these formulae are exact, it was noted, that its numerical applications are problematic when $R$ tends to unity.

A Möbius transformation of the annulus was applied in many papers to solve the Dirichlet problem for two disks (see [14,19] and a review in [10]). Constructive solution to two-particles problems in mechanics allows to describe the local interactions between particles expressed in terms of the flux around the particles. Series representations of the local fields were obtained for harmonic (heat 
and electric conduction, ideal fluids, dielectric permittivity) and biharmonic (elasticity and slow viscous fluids) equations $[8,16,26,28]$ for some shapes of particles. The most complete results for two spheres were obtained in [1] by use of the bispherical coordinates. However, the application of the obtained results to estimation of the local fields between closely spaced particles were problematic.

In the present paper, we pay attention to the conductivity problem for unidirectional cylinders stated as a $2 \mathrm{D}$ boundary value problem for the Laplace equation in a section perpendicular to cylinders. The local fields and the macroscopic conductivity properties obtained during solution to such problems are of considerable interest in the electrical or thermal conductivity, the dielectric permittivity. A review of the known results concerning the local field and the macroscopic behaviour of circular cylinders can be found in $[13,22,30]$. Besides bipolar coordinates, the method of images is frequently used to obtain an analytical solution of the boundary value problem. It is based on the successive application of the inversions with respect to circles [8]. Each inversion transforms a harmonic function in the disk to a harmonic function out of the disk and vice versa. These inversions generate the Schottky group with respect to compositions (Möbius transformations in complex variables $z$ and $\bar{z}$ ). A solution of the corresponding boundary value problem can be presented in the form of the uniformly convergent Poincaré series associated with this Schottky group [25]. The resulting Poincaré series was also written through the Schottky-Klein prime function [10,11]. Though such series theoretically converge for any multiply connected circular domain, practical computations for densely packed disks are impossible because of their too slow convergence.

Though many efforts were applied to investigate this problem in analytical form, the existing methods do not give effective analytical formulae when a small parameter $\delta$, a non-dimensional distance between the disks introduced by (2.1) below, tends to zero. For instance, the general Dirichlet problem for a doubly connected domain was solved in $[8,16,28]$, but its solution was presented in the form of a series which was not asymptotically analysed as $\delta$ tends to 0 . The simplified version of such a series can be written in the form

$$
\sum_{k=0}^{\infty}\left[(1+\sqrt{2 \delta})^{2 k}-\alpha\right]^{-1} .
$$

Figures 6-7 from [30] precisely represents that race to infinity when one attempts to approximate the considerable flux between the disks by a sum of finite order terms. The same problem of convergence for small $\delta$ arise in other two-particles problems [1].

According to [17] the electrostatic capacity of two perfectly conducting disks is proportional to $\frac{1}{\sqrt{\delta}}$ as $\delta \rightarrow 0$. Asymptotic formulae for the effective conductivity of the square and hexagonal arrays were deduced in [17]. ${ }^{1}$ It was based on the simple exact formulae for the local field around two charged cylinders in the absence of an external field (see formulae (2.7)-(2.9) in the present paper).

\footnotetext{
${ }^{1}$ see also a formula for an arbitrary square array of perfectly conducting disks in [15].
} 
This result was confirmed in [16] in terms of the energy release rates of two circular elastic inclusions under anti-plane shear deformation. The result [17] was used to get some progress in many-particles systems in $[3,4,5,6,7,18]$. The main obstacle of the further development of the structural approximation theory to the macroscopic properties of composite is absence of the exact or asymptotic formulae like (2.7)-(2.9) for inclusions in an external field. Because all the known methods, the fast multipole method [8], functional equations [30], the prime function [10,11], a finite element method [18], addition theorems with corresponding linear system of algebraic equations [24,27] (the " $z-$ " and the " $\zeta$-schemes" in the terminolofy of [9]) lead to too long series of the type (1.1). For smaller $\delta$ such a series becomes longer and blows up in the limit case $\delta=0$. An alternative approach related to applications of the elliptic functions leads to the crowding phenomenon [29] that complicates application of the elliptic functions for $\delta<0.1$ as follows from our numeric tests for $(2.16)$.

A new fast algorithm for computing the flux around non-overlapping disks was proposed in [22]. The key of the method is based on the exact solution of the boundary value problem for a doubly connected domain as the zero approximation for iterations. Such an available solution is known only for the Dirichlet problem with prescribed constant values on circles (see formula (2.7)). A new "hybrid basis scheme" was proposed in [9] based on the sums of two Laurent series of the complex flux with undetermined coefficients. The first series was the general Laurent series representation of a function analytic in a circular multiply connected domain. The second series expressed the Laurent series on a conformally transformed variable (viz. (2.3) form Section 2) which locally described the flux in the gap between the disks. It is worth noting that the structure of the resulting series from [22] coincides with the structure of the series from [9]. The series from [22] was obtained by iterations with the zero approximation (2.9) and the analogous series from [9] was obtained by the collocation method. Therefore, in order to extend the fast method [22] to general boundary value problems, first, we need to construct simple asymptotic formulae for the local fields for a two-particle problem. Such an investigation can be used in the hybrid basis scheme [9] as the basic elements in the series representations.

In this paper, following $[14,19]$ we first construct an exact solution of the modified Dirichlet problem for a doubly connected circular domain which describes the field around two perfectly conducting disks when an external flux is given at infinity. This solution is written in Section 2 in such a form that its dependence on the small gap parameter $\delta$ is explicitly presented. This makes possible to perform a precise asymptotic investigation of the field around the disks when the gap between the disks tends to zero (see Section 3). Such an asymptotic analysis yields the new analytical formula (3.17) valid for arbitrarily small $\delta$. Numerical examples demonstrate the effectiveness of the method. The exact formulae (2.16), (2.20) are extended and asymptotically analysed in Section 4 to the case when a prescribed singularity is located in the gap. In particular, the new asymptotic formula (4.6) is deduced. 


\section{Two Disks in External Field}

\subsection{Conformal mapping}

We will use the complex variable method. Let $z=x_{1}+i x_{2}$ denote a complex variable in the complex plane $\mathbb{C}$. Consider two mutually disjoint disks $\mathbb{D}_{k}=$ $\left\{z \in \mathbb{C}:\left|z-a_{k}\right|<r\right\}$. Let $D$ be the complement of the closed disks $\left|z-a_{k}\right| \leq r$ $(k=1,2)$ to the extended complex plane $\widehat{\mathbb{C}}=\mathbb{C} \cup\{\infty\}$ (see Figure 1).

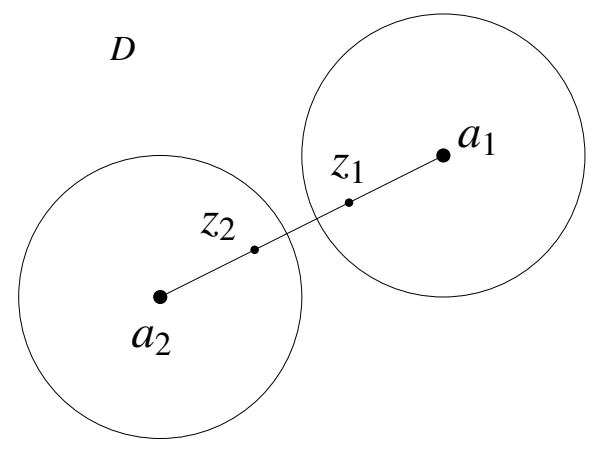

Figure 1. Two disks $\left|z-a_{k}\right|<r$ and the fixed points $z_{k}(k=1,2)$ of the map $\left(z_{(1)}^{*}\right)_{(2)}^{*}$ calculated by $(2.2)$.

The inversion of $z$ through the circle $L_{k}:=\partial \mathbb{D}_{k}$ is given by

$$
z_{(k)}^{*}=\frac{r^{2}}{z-a_{k}}+a_{k} .
$$

The Möbius transformation $\left(z_{(1)}^{*}\right)_{(2)}^{*}$ has two fixed points $z_{1}$ and $z_{2}$ satisfying the quadratic equation $z_{(1)}^{*}=z_{(2)}^{*}$. Introduce the dimensionless parameter

$$
\delta=1-\frac{2 r}{\left|a_{1}-a_{2}\right|} .
$$

If the distance $\left|a_{1}-a_{2}\right|$ tends to the minimal value $2 r$, the disks are closed to be touching, the parameter $\delta$ tends to zero. We have

$$
z_{1}=\frac{a_{1}+a_{2}}{2}+\frac{a_{1}-a_{2}}{2} \sqrt{\delta(2-\delta)}, \quad z_{2}=\frac{a_{1}+a_{2}}{2}-\frac{a_{1}-a_{2}}{2} \sqrt{\delta(2-\delta)} .
$$

One can see that $z_{k}$ belongs to $\mathbb{D}_{k}(k=1,2)$. The doubly connected domain $D$ is conformally mapped onto the annulus $D^{\prime}=\left\{z^{\prime} \in \mathbb{C}: R^{-1}<\left|z^{\prime}\right|<R\right\}$ by the function

$$
z^{\prime}=\frac{z-z_{1}}{z-z_{2}}
$$

where $R$ can be obtained by a straightforward calculation of $\left|z^{\prime}\right|$ for $z=a_{k}+r e^{i \theta}$ $(0 \leq \theta<2 \pi)$ :

$$
R=\frac{\sqrt{2-\delta}+\sqrt{\delta}}{\sqrt{2-\delta}-\sqrt{\delta}}
$$


Let us introduce the next conformal mapping

$$
w=\ln z^{\prime}=\ln \frac{z-z_{1}}{z-z_{2}}
$$

where a branch of the logarithm is chosen in such a way that the simply connected domain $D^{\prime} \backslash\left(R^{-1}, R\right)$ is mapped onto the rectangle $D^{\prime \prime}=\{w=x+i y \in$ $\mathbb{C}:|x|<\ln R,|y|<\pi\}$. It is convenient to consider the mapping $z^{\prime} \rightarrow w$ as a conformal mapping of the annulus $D^{\prime}$ onto the rectangle $D^{\prime \prime}$ with the glued together horizontal sides $x \pm \pi i(|x|<\ln R)$. Then, the circles $\left|z-a_{1}\right|=r$ and $\left|z-a_{2}\right|=r$ are transformed by (2.5) into the sides $\pm \ln R+i y(|y|<\pi)$ with the glued together points $-\ln R \pm i y$ and $-\ln R \pm i y$, respectively. The point $z=\infty$ is mapped onto the point $w=0$.

\subsection{Simple solution}

First, we discuss the well-known simple two-disks problem when the external flux is absent, i.e., the potential has not singularities in the domain $D$ and attains constant values at the boundary.

Given two constants $U_{1}$ and $U_{2}$. To find a function $u\left(x_{1}, x_{2}\right) \equiv u(z)$ harmonic in $D$ and continuous in $D \cup \partial D$ with the boundary conditions

$$
u(t)=U_{k} \quad \text { on }\left|t-a_{k}\right|=r(k=1,2) .
$$

Introduce the complex potential

$$
\varphi(z)=\frac{U_{2}-U_{1}}{2 \ln R} \ln \frac{z-z_{1}}{z-z_{2}}+\frac{U_{2}+U_{1}}{2},
$$

where a branch of the logarithm is arbitrary fixed in $\widehat{\mathbb{C}} \backslash\left[z_{1}, z_{2}\right]$ (e.g. Section 2.1 after formula (2.5)), $R$ is given by (2.4) and $z_{1,2}$ by (2.2). The well-known solution of the Dirichlet problem (2.6) is determined through the complex potential [30]

$$
u(z)=\operatorname{Re} \varphi(z)=\frac{U_{2}-U_{1}}{2 \ln R} \ln \left|\frac{z-z_{1}}{z-z_{2}}\right|+\frac{U_{2}+U_{1}}{2} .
$$

The complex flux around the disks is equal to $\overline{\varphi^{\prime}(z)}$, where the derivative

$$
\varphi^{\prime}(z)=\frac{U_{2}-U_{1}}{2 \ln R}\left(\frac{1}{z-z_{1}}-\frac{1}{z-z_{2}}\right) .
$$

Simple formulae (2.7)-(2.9) were used in [22] as the zero order approximation in the method of fast functional equations.

\subsection{Boundary value problem}

We determine the field near a pair of equal unidirectional perfectly conducting cylinders placed in a uniform applied field. The result will be not so simple as $(2.7)-(2.9)$. 
Consider the following boundary value problem (the modified Dirichlet problem)

$$
\begin{aligned}
& u=-u_{0} \quad \text { on } L_{1}, \quad u=u_{0} \quad \text { on } L_{2}, \\
& \int_{L_{k}} \frac{\partial u}{\partial n} d s=0 \quad(k=1,2)
\end{aligned}
$$

for the potential $u(z)$ harmonic in $D$ except at infinity, where $u(z) \sim x_{1}=\operatorname{Re} z$. The constant $2 u_{0}$ is equal to the jump between the values of $u(z)$ on the circles. It is undetermined and has to be found during solution to the problem (2.10)(2.11). This problem has a unique solution.

The function $u(z)$ can be considered as the real part of the function $\varphi(z)$ analytic in $D$ except at infinity, where $\varphi(z) \sim z$. It follows from $(2.11)$ that $\varphi(z)$ is single valued in $D$. The boundary condition (2.10) becomes

$$
\operatorname{Re} \varphi(z)=-u_{0} \quad \text { on } L_{1}, \quad \operatorname{Re} \varphi(z)=u_{0} \quad \text { on } L_{2} .
$$

Introduce the function $\Omega(w)=\varphi(z)$ analytic in $D^{\prime \prime}$, where $z$ and $w$ are related by $(2.5)$. The asymptotic relation $\varphi(z) \sim z$, as $z \rightarrow \infty$, becomes

$$
\Omega(w) \sim \frac{z_{2}-z_{1}}{w}=-\frac{\sqrt{\delta(2-\delta)}\left(a_{1}-a_{2}\right)}{w}, \quad \text { as } w \rightarrow 0,
$$

since $z=\left(z_{2} e^{w}-z_{1}\right)\left(e^{w}-1\right)^{-1}$. The boundary condition (2.12) becomes

$$
\begin{gathered}
\operatorname{Re} \Omega(w)=-u_{0} \quad \text { for } w=-\ln R+i y, \\
\operatorname{Re} \Omega(w)=u_{0} \quad \text { for } w=\ln R+i y(|y|<\pi) .
\end{gathered}
$$

Moreover, the glued together sides $x \pm \pi i(|x| \leq \ln R)$ yields

$$
\Omega(x-\pi i)=\Omega(x+\pi i), \quad|x| \leq \ln R .
$$

The problem (2.14)-(2.15) has the unique solution

$$
\Omega(w)=p\left[\zeta(w)-\frac{\zeta(\pi i)}{\pi i} w\right]+\frac{i q}{s n w^{\prime}}, \quad w \in D^{\prime \prime} ; \quad u_{0}=\frac{p}{2},
$$

where

$$
\begin{aligned}
& w^{\prime}=\sqrt{e_{1}-e_{3}} w, \\
& p=-\sqrt{\delta(2-\delta)} \operatorname{Re}\left(a_{1}-a_{2}\right), \quad q=-\sqrt{e_{1}-e_{3}} \sqrt{\delta(2-\delta)} \operatorname{Im}\left(a_{1}-a_{2}\right),
\end{aligned}
$$

$\zeta(w)$ denotes the $\zeta$-function of Weierstrass $s n\left(\sqrt{e_{1}-e_{3}} w\right)$ the Jacobi elliptic sinus corresponding to the periodicity cell $D^{\prime \prime}$ (see Appendix). Formula (2.16) can be justified by use of the properties of elliptic functions [2] (see also Appendix) in the following way. Let $\wp(w)$ denote the $\wp$-function of Weierstrass. First, we note that $\zeta( \pm \ln R), \wp( \pm \ln R), \wp(i y)$ are real and $\zeta(i y), \zeta(\pi i), \wp^{\prime}(i y)$ are pure imaginary since the periodicity cell is a rectangle; $\wp(z)$ is even and $\zeta(z)$ is odd. Legendre's formula (5.12) becomes

$$
\zeta(\ln R) \pi i-\zeta(\pi i) \ln R=\frac{1}{2} \pi i .
$$



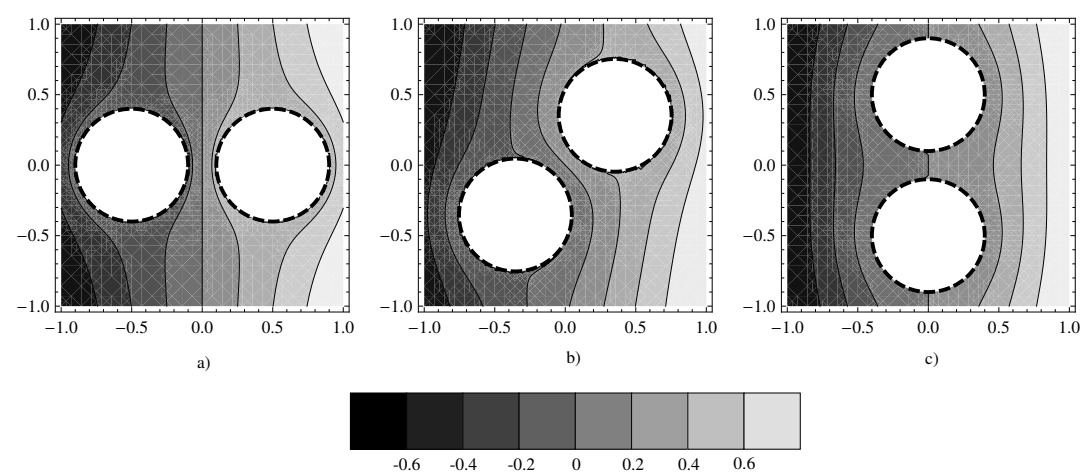

Figure 2. Two disks in the uniform external field expressed by the potential $x$ for various locations of the centers with $\delta=0.2$. Data are for the potential $\operatorname{Re} \varphi(z)$ and the constant $u_{0}$ calculated with (2.20) and (2.21): a) $\left.a_{2}=-a_{1}=\frac{1}{2}, u_{0}=0.3 ; \mathrm{b}\right)$ $a_{2}=-a_{1}=\frac{1}{2 \sqrt{2}}(1+i), u_{0}=0.212132 ;$ c) $a_{2}=-a_{1}=\frac{i}{2}, u_{0}=0$.

Using (2.19), (5.13) and formula $\wp^{\prime}( \pm \ln R)=0$ for $w= \pm \ln R+i y$ we have

$$
\begin{aligned}
& \operatorname{Re}\left[\zeta(w)-\frac{\zeta(\pi i)}{\pi i} w\right] \\
& \quad=\operatorname{Re}\left[\zeta( \pm \ln R)+\zeta(i y)+\frac{1}{2} \frac{\wp^{\prime}( \pm \ln R)-\wp^{\prime}(i y)}{\wp( \pm \ln R)-\wp(i y)} \mp \ln R \frac{\zeta(\pi i)}{\pi i}\right]= \pm \frac{1}{2}
\end{aligned}
$$

It follows from the second relation (5.4) that the function $\zeta(w)-\frac{\zeta(\pi i)}{\pi i} w$ has the period $2 \omega_{2}=2 \pi i$. The function $\operatorname{sn}\left(\sqrt{e_{1}-e_{3}} w\right)$ is double periodic, i.e., $s n\left(\sqrt{e_{1}-e_{3}}\left(w+2 \omega_{k}\right)\right)=s n\left(\sqrt{e_{1}-e_{3}} w\right)$ for $k=1,2$. Therefore, $\Omega(w)$ satisfies (2.15).

The function $\operatorname{sn}\left(\sqrt{e_{1}-e_{3}} w\right)$ maps $\partial D^{\prime \prime}$ onto the real axis. This implies that $\operatorname{Re}\left[i q\left(\operatorname{sn}\left(\sqrt{e_{1}-e_{3}} w\right)\right)^{-1}\right]=0$ for $w \in \partial D^{\prime \prime}$. Therefore, $\Omega(w)$ satisfies the boundary conditions (2.14) $-(2.15)$. The constants $p$ and $q$ in (2.18) are chosen in such a way that $\Omega(w)$ has the required asymptotic behaviour (2.13) since $\zeta(w) \sim w^{-1}$ and $\operatorname{sn}\left(\sqrt{e_{1}-e_{3}} w\right) \sim \sqrt{e_{1}-e_{3}} w$, as $w \rightarrow 0$.

Substitution of (2.5) into (2.16) yields the solution of the problem (2.12)

$$
\varphi(z)=\Omega\left(\ln \frac{z-z_{1}}{z-z_{2}}\right) .
$$

The second equation (2.16) gives the difference of the potentials on the disks

$$
u_{0}=-\frac{1}{2} \sqrt{\delta(2-\delta)} \operatorname{Re}\left(a_{1}-a_{2}\right)
$$

Formulae (2.16)-(2.18), (2.20)-(2.21) are similar to Lagally's formula (15) from [19] (see also formula (47) from [10]). All these exact formulae give correct numerical results for $\delta>0.1$. Typical contour plots of the potential (2.20) is displayed in Figure 2. For smaller $\delta$, direct numeric computations by these formulae are problematic because of the crowding phenomenon [29]. In the next section, we deduce numerically effective asymptotic formulae. 


\section{Asymptotic Formulae for Small Gap}

\subsection{Local asymptotic formulae}

Introduce the complex flux

$$
\psi(z)=\frac{\partial u}{\partial x}-i \frac{\partial u}{\partial y} \text { in } D .
$$

The function $\psi(z)$ is analytic in $D$ and $\psi(\infty)=1$. In the present section, an asymptotic formulae for $\varphi(z)$ and $\psi(z)$ are deduced as $\delta$ tends to zero and $z$ is located at the neck between the disks, i.e., near the point $\left(a_{1}+a_{2}\right) / 2$.

The flux can be approximately calculated by formula

$$
\psi(z)=\Omega^{\prime}\left(\ln \frac{z-z_{1}}{z-z_{2}}\right)\left(\frac{1}{z-z_{1}}-\frac{1}{z-z_{2}}\right) \approx \Omega^{\prime}(\pi i)\left(\frac{1}{z-z_{1}}-\frac{1}{z-z_{2}}\right)
$$

near the middle point $z=\left(a_{1}+a_{2}\right) / 2$ since $(2.2)$ implies that

$$
\ln \frac{\left(a_{1}+a_{2}\right) / 2-z_{1}}{\left(a_{1}+a_{2}\right) / 2-z_{2}}=\ln (-1)=\pi i .
$$

The derivative of the function (2.16) has the form (see (5.10))

$$
\Omega^{\prime}(w)=-p\left[\wp(w)+\frac{\zeta(\pi i)}{\pi i}\right]-\frac{i q \sqrt{e_{1}-e_{3}}}{s n^{2} w^{\prime}} \sqrt{\left(1-s n w^{\prime}\right)\left(1-k^{2} s n w^{\prime}\right)},
$$

where $w^{\prime}$ is given by $(2.17)$.

We are interested in the asymptotic behaviour of the values (5.5)-(5.11) when $w \rightarrow \pi i(\delta \rightarrow 0)$ to estimate the flux between the disks by (3.1). The half-periods of the elliptic functions have the form $\omega_{1}=\ln R$ and $\omega_{2}=\pi i$ where $R$ is given by (2.4). We have

$$
\ln R=\ln \frac{1+\sqrt{\frac{\delta}{2-\delta}}}{1-\sqrt{\frac{\delta}{2-\delta}}}=\sqrt{2 \delta}+O\left(\delta^{3 / 2}\right), \quad \text { as } \delta \rightarrow 0 .
$$

Introduce the constant (compare to (5.8))

$$
h=\exp \left(-\frac{\pi^{2}}{\ln R}\right) .
$$

It follows form (3.3) and (3.4) that $h$ can be approximated by an exponentially small value denoted by $o(\delta)$

$$
h \approx \exp \left(-\frac{\pi^{2}}{\sqrt{2 \delta}}\right)=o(\delta), \quad \text { as } \delta \rightarrow 0 .
$$

Here, $o(\delta)$ is defined as a value less than any positive power of $\delta$, i.e.,

$$
\lim _{\delta \rightarrow 0+} \frac{o(\delta)}{\delta^{N}}=0 \quad \text { for any positive } N .
$$


We now proceed to estimate $\eta_{1}$ and $\eta_{2}$ (see (5.1)). It follows from (3.3) and (3.5) that

$$
\eta_{1}=\frac{\pi^{2}}{12 \sqrt{2 \delta}}+o(\delta)
$$

The value $\eta_{2}=\zeta(\pi i)$ can be found from Legendre's identity (5.12) (see also $(2.19))$

$$
\eta_{2}=\pi i\left(\frac{\pi^{2}}{24 \delta}-\frac{1}{2 \sqrt{\delta(2-\delta)}}\right)+o(\delta) .
$$

Then, the constants (5.5)-(5.7) can be estimated as follows

$$
e_{1} \approx \frac{\pi^{2}}{12 \delta}+o(\delta), \quad e_{2} \approx-\frac{\pi^{2}}{24 \delta}+\frac{\pi^{2}}{\delta} h, \quad e_{3} \approx-\frac{\pi^{2}}{24 \delta}-\frac{\pi^{2}}{\delta} h,
$$

where the approximations are valid up to $O\left(h^{2}\right)$. Hence,

$$
e_{1}-e_{3} \approx \frac{\pi^{2}}{\delta}\left(\frac{1}{8}+h\right), \quad e_{2}-e_{3} \approx \frac{2 \pi^{2}}{\delta} h
$$

and (5.11) yields

$$
k \approx 4 \exp \left(-\frac{\pi^{2}}{2 \sqrt{2 \delta}}\right) .
$$

Application of (5.7), (3.9) and (3.8) yields

$$
\wp(\pi i)+\frac{\zeta(\pi i)}{\pi i}=e_{3}+\frac{\eta_{2}}{\omega_{2}}=-\frac{1}{2 \sqrt{\delta(2-\delta)}}+o(\delta) .
$$

It follows from $\left(s n w^{\prime}\right)^{-2} \rightarrow 0$, as $w \rightarrow \pi i$, that

$$
\lim _{w \rightarrow \pi i} \sqrt{\left(1-\frac{1}{s n w^{\prime}}\right)\left(k^{2}-\frac{1}{s n w^{\prime}}\right)}=k,
$$

where $k$ has the exponentially small order (3.10). Hence, (3.2) and (2.18) yield

$$
\Omega^{\prime}(\pi i)=\frac{1}{2} \operatorname{Re}\left(a_{1}-a_{2}\right)+o(\delta) .
$$

Therefore,

$$
\varphi^{\prime}(z) \approx \frac{\operatorname{Re}\left(a_{1}-a_{2}\right)}{2}\left(\frac{1}{z-z_{1}}-\frac{1}{z-z_{2}}\right)=: \Psi(z) .
$$

Introduce the function

$$
\Phi(z)=\frac{\operatorname{Re}\left(a_{1}-a_{2}\right)}{2} \ln \frac{z-z_{1}}{z-z_{2}}
$$

satisfying $\Psi(z)=\Phi^{\prime}(z)$. The function $u(z)=\operatorname{Re} \Phi(z)$ solves the Dirichlet problem (2.6) with

$$
U_{1}=-\frac{\operatorname{Re}\left(a_{1}-a_{2}\right)}{2} \ln R, \quad U_{2}=\frac{\operatorname{Re}\left(a_{1}-a_{2}\right)}{2} \ln R .
$$


It is worth noting that $\Phi(z)$ vanishes at infinity and it is not single-valued in $D$ contrary to $\varphi(z)$ from (2.20) satisfying the problem (2.12).

Formula (3.11) describes asymptotically the flux between two equal disks with the centres at $a_{1}$ and $a_{2}$ when $\delta$ tends to zero. It is worth noting that the obtained asymptotics coincides with the result of [30] and [18]. The constant multiplier in (3.11) is written up to an exponentially small term $o(\delta)$, as $\delta$ tends to zero.

\subsection{Global asymptotic formulae}

In the present section, we construct an approximation of (2.20) asymptotically valid in the whole domain $D$ for sufficiently small $\delta$. This approximation is based on the degenerate elliptic functions discussed in [2].

Each term $h^{2 k} \exp \left( \pm \frac{\pi w}{\omega_{1}}\right)$ from (5.1) can be estimated by use of (3.5) and Im $w \leq \pi$ as follows

$$
\left|h^{2 k} \exp \left( \pm \frac{\pi w}{\omega_{1}}\right)\right| \leq \exp \left(-\frac{2 k \pi^{2}}{\sqrt{2 \delta}}\right) \exp \left(\frac{\pi^{2}}{\sqrt{2 \delta}}\right) \leq \exp \left(-\frac{\pi^{2}}{\sqrt{2 \delta}}\right) .
$$

Therefore, (5.2) and (3.7) yield

$$
\zeta(w)=\frac{\pi^{2}}{24 \delta} w+\frac{\pi}{2 \sqrt{2 \delta}} \cot \frac{\pi w}{2 \sqrt{2 \delta}}+o(\delta) .
$$

Here, the estimation in $\delta$ is not uniform in $w$. The Weierstrass function $\wp(w)$ can be analogously estimated by use of (5.3)

$$
\wp(w)=-\frac{\pi^{2}}{24 \delta}+\frac{\pi^{2}}{8 \delta} \frac{1}{\sin ^{2} \frac{\pi w}{2 \sqrt{2 \delta}}}+o(\delta) .
$$

Estimations (3.9) and formula (5.9) yield

$$
\frac{1}{s n w^{\prime}}=\frac{1}{\sin w^{\prime}}+o(\delta) \quad \text { with } w^{\prime}=\frac{\pi w}{2 \sqrt{2 \delta}}+o(\delta) .
$$

Substitute (3.14), (3.16), (3.8) into (2.16), (2.20) and take terms up to $O(\delta)$

$$
\varphi(z)=-\frac{\operatorname{Re}\left(a_{1}-a_{2}\right)}{2}\left(\pi \cot w^{\prime}+\ln \frac{z-z_{1}}{z-z_{2}}\right)-\frac{i \operatorname{Im}\left(a_{1}-a_{2}\right)}{2} \frac{\pi}{\sin w^{\prime}}+O(\delta),
$$

where

$$
w^{\prime}=\frac{\pi}{2 \sqrt{2 \delta}} \ln \frac{z-z_{1}}{z-z_{2}}+o(\delta), \quad z \in D .
$$

(3.17)-(3.18) give the desired global asymptotic formula for the complex potential.

\section{Singularity in the Gap}

In Sections 2-3, the uniform external field is modelled by the principal part $z$ of the function $\varphi(z)$ at infinity, i.e., $\varphi(z)$ is analytic in $D$ except at infinity where it 
has a pole. The location of singularities and their types can be arbitrary in the domain $D$. In the present section, we consider a numerically difficult example when a singularity is located in the gap between the disks. More precisely, it is assumed that the function $\varphi(z)$ is analytic in $D$ except at infinity and at $z=\frac{1}{2}\left(a_{1}+a_{2}\right)$ where its principal parts are given by the asymptotic formulae

$$
\varphi(z) \sim z, \quad z \rightarrow \infty ; \quad \varphi(z) \sim \frac{\alpha}{z-\frac{1}{2}\left(a_{1}+a_{2}\right)}, \quad z \rightarrow \frac{1}{2}\left(a_{1}+a_{2}\right),
$$

where the given constant $\alpha$ stands for the intensity of the considered dipole at $z=\frac{1}{2}\left(a_{1}+a_{2}\right)$.

Following Section 2 we are looking for the complex potential $\varphi(z)$ satisfying the boundary conditions (2.12). Then the function $\Omega(w)=\varphi(z)$ is analytic in $D^{\prime \prime}$. The asymptotic relations (4.1) become

$$
\Omega(w) \sim \frac{\sqrt{\delta(2-\delta)}\left(a_{2}-a_{1}\right)}{w}, \quad w \rightarrow 0 ; \quad \Omega(w) \sim \frac{\beta}{w-\pi i}, \quad w \rightarrow \pi i,
$$

where $\beta=4 \alpha /\left(\left(a_{2}-a_{1}\right) \sqrt{\delta(2-\delta)}\right)$. The function $\Omega(w)$ satisfies the same boundary conditions $(2.14)-(2.15)$. Instead of the representation $(2.16) \Omega(w)$ has the form

$$
\begin{aligned}
& \Omega(w)=\sqrt{\delta(2-\delta)} \operatorname{Re}\left(a_{2}-a_{1}\right) \zeta(w)+\frac{4}{\sqrt{\delta(2-\delta)}} \operatorname{Re} \frac{\alpha}{a_{2}-a_{1}} \zeta(w-\pi i)-2 u_{0} \frac{\eta_{2}}{\pi i} w \\
& \quad+2 i \sqrt{e_{1}-e_{3}}\left[\frac{\gamma_{1}}{s n\left(\sqrt{e_{1}-e_{3}} w\right)}+\frac{\gamma_{2}}{s n\left(\sqrt{e_{1}-e_{3}}(w-\pi i)\right)}\right], w \in D^{\prime \prime}
\end{aligned}
$$

and

$$
u_{0}=\frac{1}{2} \sqrt{\delta(2-\delta)} \operatorname{Re}\left(a_{2}-a_{1}\right)+\frac{2}{\sqrt{\delta(2-\delta)}} \operatorname{Re} \frac{\alpha}{a_{2}-a_{1}}
$$

where

$$
\gamma_{1}=\frac{1}{2} \sqrt{\delta(2-\delta)} \operatorname{Im}\left(a_{2}-a_{1}\right), \gamma_{2}=\frac{2}{\sqrt{\delta(2-\delta)}} \operatorname{Im} \frac{\alpha}{a_{2}-a_{1}} .
$$

The proof of (4.3)-(4.5) is based on the comparison of the principal parts of $\Omega(w)$ from (4.2), (4.3) and follows the justification of (2.16) from Section 2.3.

Following Section 3.2 we transform the exact formula (4.3) by an asymptotic expression. Application of (3.14)-(3.16) yields

$$
\begin{aligned}
\Omega(w)= & \frac{\operatorname{Re}\left(a_{2}-a_{1}\right)}{2} \pi \cot \frac{\pi w}{2 \sqrt{2 \delta}}+\frac{\pi}{2 \delta} \operatorname{Re} \frac{\alpha}{a_{2}-a_{1}} \cot \frac{\pi(w-\pi i)}{2 \sqrt{2 \delta}} \\
& -\frac{\pi^{2}}{6 \delta \sqrt{2 \delta}} \operatorname{Re} \frac{\alpha}{a_{2}-a_{1}}(w+\pi i) \\
& +\frac{\pi i}{\sqrt{2 \delta}}\left\{\frac{\gamma_{1}}{\sin \frac{\pi w}{2 \sqrt{2 \delta}}}+\frac{\gamma_{2}}{\sin \frac{\pi(w-\pi i)}{2 \sqrt{2 \delta}}}\right\}+O\left(\delta^{0}\right), \quad w \in D^{\prime \prime} .
\end{aligned}
$$

The complex potential $\varphi(z)$ has the form (2.20). The flux is calculated through the derivative $\frac{d}{d z} \varphi(z)$. 

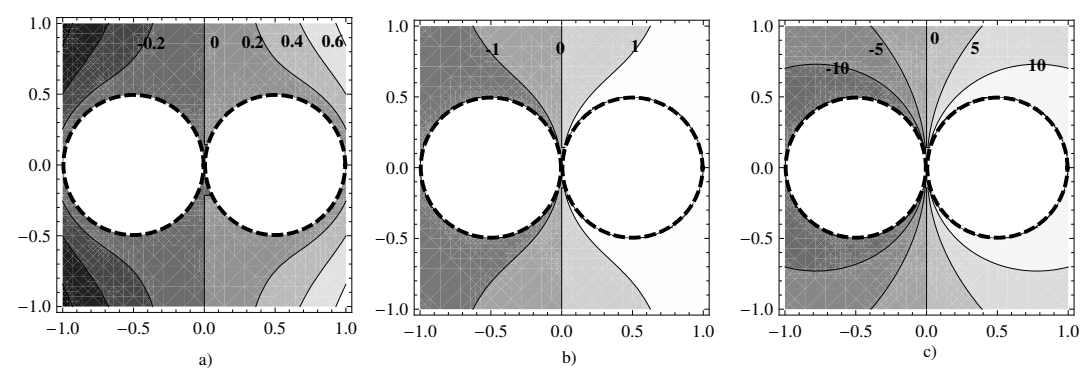

Figure 3. Modulus of the flux in the domain with $a_{2}=-a_{1}=0.5, \delta=0.01$ in the presence of the external flux $(1,0)$ and the dipole $\frac{\alpha}{z}$ of intensity $\alpha$. Data and $u_{0}$ are for: a) $\alpha=0, u_{0}=0.070534$; b) $\alpha=0.1, u_{0}=1.488296$; c) $\alpha=1, u_{0}=14.24816$. Labels to contour lines are written.

Formula (4.6) is effective in computations for $\delta<0.1$. The intensity of flux $\left|\frac{d}{d z} \varphi(z)\right|$ is displayed in Figure 3 for different intensities of the dipole. Figure 3a shows the flux when the dipole is absent. In the next figures the intensity of the dipole increases that locally change the flux near the gap.

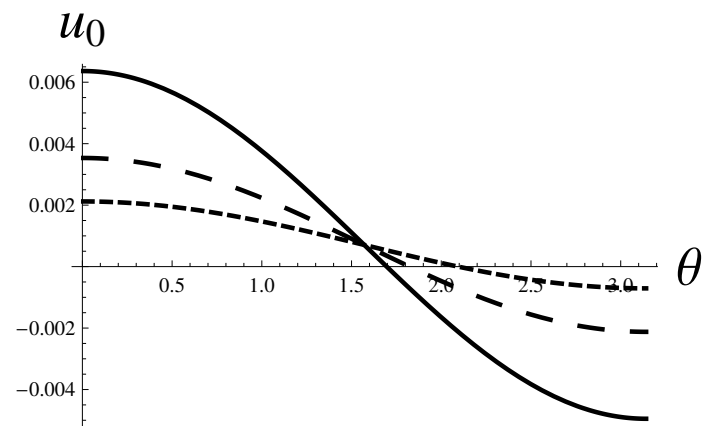

Figure 4. Dependence of $u_{0}$ from (4.4) on the intensity $|\alpha|$ and the direction $\theta$ of the dipole $(0 \leq \theta<\pi)$ when $\alpha=|\alpha| e^{i \theta}$ for $a_{2}=-a_{1}=0.5, \delta=10^{-6}$. Data are for: $\alpha=0.410^{-5}$ (solid line); $\alpha=0.210^{-5}$ (long dashed line); $\alpha=10^{-6}$ (short dashed line).

Consider an example when the intensity of dipole has the form $\alpha=|\alpha| e^{i \theta}$ $(0 \leq \theta<2 \pi)$, i.e., the amplitude of the dipole has the intensity $|\alpha|$ and its direction is equal to $\theta$. The dependence of $u_{0}$ from (4.4), the difference of the potential on the boundary circles, on $\alpha$ is shown in Figure 4 . All the plots in Figure 4 has the same value $u_{0}=0.00070610$ at $\theta=\frac{\pi}{2}$. The common value of $u_{0}$ changes with $\delta$.

\section{Discussion}

Fields around two particles embedded in a two-dimensional background were investigated by various methods. The local fields were obtained in the form of series by the classic method of images and its extensions $[8,10,11,16,20$, 
$21,23,27,30]$. For closely placed inclusions, the previous methods yield slowly convergent series. An exception is the Dirichlet problem (2.6) whose solution is given by simple formulae (2.7)-(2.9). The latter formulae were applied in [22] as the a zeroth approximation to a fast method for many inclusions.

An alternative approach based on the exact solution to the Dirichlet problem in terms of the elliptic functions is presented in the classical works $[12$, $14,19,31]$. However, for smaller $\delta$, direct numeric computations by these formulae require too much computational resources because of the crowding phenomenon [29].

Asymptotic formulae (3.11)-(3.13), (3.17)-(3.18) deduced in this paper give accurate numeric results for arbitrary small $\delta$ and have a simple structure. These formulae can be applied to the fast method for many inclusions [22] and can be used in the hybrid basis scheme [9] as the basic elements in the series representations. The main advantage of the deduced asymptotic formulae is based on the exactly written main asymptotic term proportional to $\frac{1}{\sqrt{\delta}}$.

The exact formula is extended to the case when a prescribed singularity is located in the gap between the disks (see formulae (4.3)-(4.5)). This case is difficult in numerical realizations for small $\delta$ by the method of images because the distance $\left(a_{2}-a_{1}\right) \sqrt{\delta(2-\delta)}$ between the points $z_{1}$ and $z_{2}$ accumulating singularities is small. Pure numerical methods are also problematic for such a problem. Our asymptotic formula (4.6) as well as (3.17)-(3.18) have the same numerical limitations on $\delta$ as the trigonometric functions from (4.6), hence, practically have not. This extends applications of structural approximations $[7,18]$ to estimations of the local fields in densely packed fiber composites in various external fields.

\section{Acknowledgement}

I am grateful to Darren Crowdy for the invitation to the AMMP Workshop "Conformal geometry in mapping, imaging and sensing" (2013), to Vladimir Mityushev and Sergei Rogosin for the discussion following this workshop, to Igor Andrianov and Galina Starushenko for useful remarks.

\section{Appendix}

In this appendix, some formulae and properties of the elliptic function theory are presented in accordance with [2]. Let $\omega_{1}$ and $\omega_{2}$ denote the half-periods of the periodicity cell; $\zeta(w)$ and $\wp(z)=-\zeta^{\prime}(w)$ the Weierstrass functions; sn $w$ the Jacobi elliptic sinus. The function $\zeta(w)$ can be expanded into Laurent's series

$$
\zeta(w)=\frac{1}{w}-c_{3} w^{3}-c_{5} w^{5}-\cdots
$$

with some constants $c_{j}$. Introduce the constants $\eta_{k}=\zeta\left(\omega_{k}\right)(k=1,2)$. We have

$$
2 \eta_{1} \omega_{1}=\frac{\pi^{2}}{6}\left[1-24 \sum_{k=1}^{\infty} \frac{h^{2 k}}{\left(1-h^{2 k}\right)^{2}}\right],
$$




$$
\begin{aligned}
\zeta(w)= & \frac{\eta_{1}}{\omega_{1}} w+\frac{\pi}{2 \omega_{1}}\left[\cot \frac{\pi w}{2 \omega_{1}}\right. \\
& \left.+i \sum_{k=1}^{\infty}\left(\frac{2 h^{2 k} \exp \left(-\frac{\pi i w}{\omega_{1}}\right)}{1-h^{2 k} \exp \left(-\frac{\pi i w}{\omega_{1}}\right)}-\frac{2 h^{2 k} \exp \left(\frac{\pi i w}{\omega_{1}}\right)}{1-h^{2 k} \exp \left(\frac{\pi i w}{\omega_{1}}\right)}\right)\right]
\end{aligned}
$$

and

$$
\begin{aligned}
\wp(w)= & -\frac{\eta_{1}}{\omega_{1}}+\left(\frac{\pi}{2 \omega_{1}}\right)^{2}\left[\frac{1}{\sin ^{2} \frac{\pi w}{2 \omega_{1}}}\right. \\
& \left.-4 \sum_{k=1}^{\infty}\left(\frac{h^{2 k} \exp \left(-\frac{\pi i w}{\omega_{1}}\right)}{\left(1-h^{2 k} \exp \left(-\frac{\pi i w}{\omega_{1}}\right)\right)^{2}}+\frac{h^{2 k} \exp \left(\frac{\pi i w}{\omega_{1}}\right)}{\left(1-h^{2 k} \exp \left(\frac{\pi i w}{\omega_{1}}\right)\right)^{2}}\right)\right] .
\end{aligned}
$$

The function $\zeta(w)$ has the constant increments on the periodicity cell

$$
\zeta\left(w+2 \omega_{1}\right)-\zeta(w)=2 \eta_{1}, \quad \zeta\left(w+2 \omega_{2}\right)-\zeta(w)=2 \eta_{2} .
$$

The function $\wp(w)$ is double periodic.

The following constants are important in the theory of elliptic functions

$$
\begin{aligned}
& e_{1}=\wp\left(\omega_{1}\right)=-\frac{\eta_{1}}{\omega_{1}}+\left(\frac{\pi}{\omega_{1}}\right)^{2}\left[\frac{1}{4}+2 \sum_{k=1}^{\infty} \frac{h^{2 k}}{\left(1+h^{2 k}\right)^{2}}\right], \\
& e_{2}=\wp\left(\omega_{1}+\omega_{2}\right)=-\frac{\eta_{1}}{\omega_{1}}+2\left(\frac{\pi}{\omega_{1}}\right)^{2} \sum_{k=1}^{\infty} \frac{h^{2 k-1}}{\left(1+h^{2 k-1}\right)^{2}}, \\
& e_{3}=\wp\left(\omega_{2}\right)=-\frac{\eta_{1}}{\omega_{1}}-2\left(\frac{\pi}{\omega_{1}}\right)^{2} \sum_{k=1}^{\infty} \frac{h^{2 k-1}}{\left(1-h^{2 k-1}\right)^{2}},
\end{aligned}
$$

where

$$
h=\exp \left(\pi i \frac{\omega_{2}}{\omega_{1}}\right) .
$$

The Jacobi elliptic sinus can be calculated via the Weierstrass $\wp$-function

$$
\operatorname{sn}^{2} w^{\prime}=\frac{e_{1}-e_{3}}{\wp(w)-e_{3}}, \quad w^{\prime}=\sqrt{e_{1}-e_{3}} w .
$$

It follows from (5.5), (5.7) and (5.8) that the difference $e_{1}-e_{3}$ is always positive for rectangular periodicity cells $\left(\omega_{1}>0\right.$ and $\left.i \omega_{2}<0\right)$. The derivative $\frac{d}{d w^{\prime}} \operatorname{sn} w^{\prime}$ can be expressed by sn $w$ as follows

$$
\frac{d}{d w^{\prime}} \operatorname{sn} w^{\prime}=\sqrt{\left(1-\operatorname{sn} w^{\prime}\right)\left(1-k^{2} \operatorname{sn} w^{\prime}\right)}
$$

where

$$
k^{2}=\frac{e_{2}-e_{3}}{e_{1}-e_{3}} .
$$

Legendre's identity has the form

$$
\eta_{1} \omega_{2}-\eta_{2} \omega_{1}=\frac{\pi i}{2}
$$

The following formula is used in verification of (2.16)

$$
\zeta(u+v)=\zeta(u)+\zeta(v)+\frac{1}{2} \frac{\wp^{\prime}(u)-\wp^{\prime}(v)}{\wp(u)-\wp(v)} .
$$




\section{References}

[1] P.M. Adler. Interaction of unequal spheres. II-Conducting spheres. J. Coll. Int. Sc., 84:475-488, 1981. http://dx.doi.org/10.1016/0021-9797(81)90238-1.

[2] N.I. Akhiezer. Elements of the Theory of Elliptic Functions. American Mathematical Society, 1990.

[3] I.V. Andrianov, V.V. Danishevs'kyy, A. Guillet and Ph. Pareige. Effective properties and micro-mechanical response of filamentary composite wires under longitudinal shear. Eur. J. Mech. A Solids, 24:195-206, 2005. http://dx.doi.org/10.1016/j.euromechsol.2005.01.006.

[4] I.V. Andrianov, V.V. Danishevskyy and D. Weichert. Simple estimations on effective transport properties of a random composite material with cylindrical fibres. ZAMP, 2:889-903, 2008. http://dx.doi.org/10.1007/s00033-007-6146-3.

[5] I.V. Andrianov, A.L. Kalamkarov and G.A. Starushenko. Analytical expressions for effective thermal conductivity of composite materials with inclusions of square cross-section. Composites Part B: Engineering, 50:44-53, 2013. http://dx.doi.org/10.1016/j.compositesb.2013.01.023.

[6] I.V. Andrianov, A.L. Kalamkarov and G.A. Starushenko. Three-phase model for a fiber-reinforced composite material. Composite Structures, 95:95-104, 2013. http://dx.doi.org/10.1016/j.compstruct.2012.07.003.

[7] L. Berlyand, A. Kolpakov and S. Novikov. Introduction to the Network Approximation Method for Materials Modeling. Cambridge University Press, Cambridge, 2012 .

[8] H. Cheng and L. Greengard. A method of images for the evaluation of electrostatic fields in systems of closely spaced conducting cylinders. SIAM J. Appl. Math., 58:122-141, 1998. http://dx.doi.org/10.1137/S0036139996297614.

[9] D. Crowdy, S. Tanveer and T. DeLillo. Hybrid basis scheme for computing electrostatic fields exterior to close-to-touching discs. arXiv:1409.2466, 2014.

[10] D.G. Crowdy. Analytical solutions for uniform potential flow past multiple cylinders. Eur. J. Mech. B Fluids, 25:459-470, 2006. http://dx.doi.org/10.1016/j.euromechflu.2005.11.005.

[11] D.G. Crowdy. The Schottky-Klein prime function on the Schottky double of planar domains. Comp. Meth. Funct. Theory, 10:501-517, 2010. http://dx.doi.org/10.1007/BF03321778.

[12] U. Dini. Il problema di Dirichlet in unarea anulare, e nello spazio compreso fra due sfere concentriche. Rend. Circ. Mat. Palermo, 36:1-28, 1913. http://dx.doi.org/10.1007/BF03016009.

[13] M.V. Dubatovskaya and S.V. Rogosin. On effective conductivity formula for bounded symmetric composite material. In T. Aliev Azeroglu, A. Golberg and S. Rogosin(Eds.), Complex Analysis and Potential Theory with Applications, pp. 53-62, Cambridge, 2014. Cambridge Scientific Publishers.

[14] C. Ferrari. Sulla trasformazione conforme di due cerchi in due profili alari, Memorie della. Reale Accademia delle Scienze di Torino, Serie II, 67:1-15, 1930 .

[15] S. Gluzman, V. Mityushev and W. Nawalaniec. Cross-properties of the effective conductivity of the regular array of ideal conductors. Arch. Mech., 66:287-301, 2014 . 
[16] E. Honein, T. Honein and G. Herrmann. Energetics of two circular inclusions in anti-plane elastostatics. Internat. J. Solids Structures, 37:3667-3679, 2000. http://dx.doi.org/10.1016/S0020-7683(98)00286-8.

[17] J.B. Keller. Conductivity of a medium containing a dense array of perfectly conducting spheres or cylinders or nonconducting cylinders. J. Appl. Phys., 34:991-993, 1963. http://dx.doi.org/10.1063/1.1729580.

[18] A.A. Kolpakov and A.G. Kolpakov. Capacity and Transport in Contrast Composite Structures: Asymptotic Analysis and Applications. CRC Press Inc., Boca Raton etc., 2009.

[19] M. Lagally. Die reibungslose Strömung im Aussengebiet zweier Kreise. Z. Angew. Math. Mech., 9:299-305, 1929. http://dx.doi.org/10.1002/zamm.19290090405.

[20] R.C. McPhedran. Transport properties of cylinder pairs and of the square array of cylinders. Proc. R. Soc. Lond. Ser. A, 408:31-43, 1986.

[21] R.C. McPhedran and G.W. Milton. Transport properties of touching cylinder pairs and of the square array of touching cylinders. Proc. R. Soc. Lond. Ser. A, 411:313-326, 1987.

[22] V. Mityushev and N. Rylko. A fast algorithm for computing the flux around non-overlapping disks on the plane. Math. Comput. Modelling, 57:1350-1359, 2013. http://dx.doi.org/10.1016/j.mcm.2012.11.019.

[23] V.V. Mityushev. Plane problem for the steady heat conduction of material with circular inclusions. Arch. Mech., 45:211-215, 1993.

[24] V.V. Mityushev. Generalized method of Schwarz and addition theorems in mechanics of materials containing cavities. Arch. Mech., 47:1169-1181, 1995.

[25] V.V. Mityushev. Riemann-hilbert problems for multiply connected domains and circular slit maps. Comp. Meth. Funct. Theory, 11:575-590, 2011. http://dx.doi.org/10.1007/BF03321876.

[26] V.V. Mityushev, Yu. Obnosov, E. Pesetskaya and S.V. Rogosin. Analytical methods for heat conduction in composites. Math. Model. Anal., 13:67-78, 2008. http://dx.doi.org/10.3846/1392-6292.2008.13.67-78.

[27] A.B. Movchan, N.A. Nicorovici and R.C. McPhedran. Green's tensors and lattice sums for elastostatics and elastodynamics. Proc. R. Soc. Lond. Ser. A, 453:643662, 1997.

[28] Yu.V. Obnosov. Solution of a problem of a seepage fields distribution into infinite porous massif with two circular inclusions. Uch. Zap. Kazan. Gos. Univ., Ser. Fiz.-Mat. Nauk, 148:109-123, 2006.

[29] N. Papamichael and N. Stylianopoulos. Numerical Conformal Mapping: Domain Decomposition and the Mapping of Quadrilaterals. World Scientific, Hackensack, NJ, 2010.

[30] N. Rylko. Structure of the scalar field around unidirectional circular cylinders. Proc. R. Soc. Lond. Ser. A, 464:391-407, 2008.

[31] H. Villat. Le probleme de Dirichlet dans une aire annulaire. Rend. Circ. Mat. Palermo, 33:134-174, 1912. http://dx.doi.org/10.1007/BF03015296. 